\title{
ON THE EXISTENCE OF NORMAL MODE VIBRATIONS IN NONLINEAR SYSTEMS*
}

\author{
BY
}

\author{
C. H. PAK AND R. M. ROSENBERG \\ University of California, Berkeley
}

Introduction. Certain properties of normal mode vibrations have been studied fairly extensively in recent times [1]. However, the question of their existence has largely remained open (except for the case that the normal mode vibrations are "similar". This is the name applied to normal mode vibrations $x_{i}(t) / x_{i}(t) \equiv c_{i j}=$ const, $(i, j=$ $1,2, \cdots)$; i.e. where the wave shapes are similar in the sense of plane geometry). The first significant advance in establishing existence theorems for cases including nonsimilar normal mode vibrations was made recently in a paper by Cooke and Struble [2] in which the motion is, as the authors say, "near to linearized motion". This statement implies actually two properties, one referring to the structure of the system, and the other to its motion. It implies, in fact, that the system is "linearizable" [1] and that the motions are small. Under these assumptions, one can readily show that the equations of motion used by Cooke and Struble can always be modelled by a straight, anchored chain of elastically coupled particles, each possessing a single translational degree of freedom in the direction of the chain. For instance, a space array of elastically coupled particles each having three translational degrees of freedom cannot, in general, be a model for their equations because that system may be nonlinearizable, owing to the so-called "kinematic" nonlinearities [3]. Also, strongly nonlinear systems do not fall within the compass of the work by Cooke and Struble. It is probably for this reason that these authors conclude that the general question of the existence of normal mode vibrations "remains an unanswered... mathematical problem".

In this paper we demonstrate the existence of normal mode vibrations of elastically coupled, nonlinear systems where neither the system nor its motion need lie near the linear case. In fact, the systems admitted here need not have isolated equilibrium positions; they may be strongly nonlinear or nonlinearizable, and the nonlinearities may be "elastic" or "kinematic"; hence, the physically interesting space array of particles is admitted. The methods used in the existence proof are purely geometrical. They depend on the construction of a metric Riemann space on which one can utilize all theorems of Riemannian geometry; in this way, the existence proof is reduced to demonstrating the existence of certain extremal arcs in a Riemann space.

The system. A Hamiltonian system is called simple if it is conservative, holonomic and scleronomic. We consider a simple Hamiltonian system having a finite number $n$ of degrees of freedom. The generalized coordinates are the components $q_{i}$ of the vector $q=\left(q_{1}, q_{2}, \cdots, q_{n}\right)$, and the velocity vector is $\dot{q}=\left(\dot{q}_{1}, \dot{q}_{2}, \cdots, \dot{q}_{n}\right)$. Since the system is simple, the energy integral

$$
T(\dot{q})-U(q)=h, \quad t \geq 0
$$

*Received May 1, 1967; revised manuscript received October 3, 1967. 
exists, where $T$ is the kinetic energy, $U$ is the potential function, and $h=$ const is the total energy of any given motion of the system. The kinetic energy is positive definite, quadratic in the $\dot{q}_{i}$ and has a constant matrix called the inertia matrix. When that matrix is not diagonal, the system is said to be inertia-coupled. But then there always exists an orthogonal transformation which diagonalizes the inertia matrix. Hence, we may assume without loss of generality that the system is inertia-decoupled, and we denote by $m_{\boldsymbol{i}}$ the diagonal elements of the inertia matrix. Then, the energy integral is of the form

$$
\sum_{i=1}^{n} \frac{1}{2} m_{i} \dot{q}_{i}^{2}-U(q)=h .
$$

The quantities $m_{i}$ may be regarded as the masses of particles, associated with the $q_{i}$. Evidently, the $m_{i}$ need not be distinct; for instance, in a space array of particles, $n / 3$ is an integer, and for each three $q_{i}$, the $m_{i}$ are equal.

The set $\left\{q_{i}\left(t_{0}\right)\right\}$ is called the configuration of the system at the time $t_{0}$, and the Euclidean $n$-space $E^{n}$ contains the configuration space $\Phi(h)$ for a motion of energy level $h$. As the system moves, it passes through a sequence of configurations, and the locus of the points in $\Phi$, corresponding to this sequence is called the trajectory of this motion.

The motion of the system satisfies on any time interval $\left[t_{1}, t_{2}\right]$ Hamilton's principle

$$
\delta \int_{t_{1}}^{t_{*}} L d t=0
$$

where the Lagrangian function is $L=T+U$, and a necessary condition for satisfying Hamilton's principle is that every $q_{i}$ satisfy

$$
m_{i} \ddot{q}_{i}=\partial U / \partial q_{i}, \quad(i=1,2, \cdots, n) .
$$

Clearly, the nonlinearities in (2) arise from the structure of the potential function only.

The potential function is subject to all of the following restrictions, and only to them:

(i) $U(q)$ is of class $C^{3}$, and at the origin $U^{I}+h>0$;

(ii) $U(q)=U(-q)$;

(iii) the set

$$
\Gamma=\{q: U(q)+h=0\}
$$

is a closed, connected hypersurface in $E^{n}$ which surrounds the origin, ${ }^{1}$ and the gradient vector $\nabla U$ does not vanish on $\Gamma$. These conditions will be called the admissibility conditions.

The smoothness condition (i) is required in order to apply the theorems of the calculus of variations [4].

The symmetry condition (ii) holds for any system of particles interconnected by springs when the spring forces are the only potential forces of the system. It states that the interacting forces between any two particles depend only on the distance between these particles, but not on the direction of their relative position vector. The symmetry condition has the consequence that the origin of $E^{n}$ is always an equilibrium position because one has

$$
\nabla U(q)=-\nabla U(-q)
$$

${ }^{1}$ See the definitions of $\Phi(h)$ and $\Theta(h)$ in (3) and (6). 
and, thus, from the smoothness condition

$$
\nabla U(+0)=\nabla U(-0)
$$

In addition to the origin, there may be any number of (including infinitely many) isolated or neighboring other equilibrium positions. The case of neighboring equilibrium positions may arise in cases of physical interest-for instance, when an equilibrium position is "neutral".

The stagnation condition (iii) defines the locus of rest points or stagnation configurations because the kinetic energy vanishes on $\Gamma$. It follows from (1) that the trajectories of all motions with energy level $h$ are bounded by $\Gamma$; therefore, $\Gamma$ will also be called the bounding surface. One observes that, because of the energy integral (i) and because of (iii), $U$ takes on its largest absolute value on $\Gamma$.

Under the nonsingular transformations

$$
x_{i}=\left(m_{i}\right)^{1 / 2} q_{i}, \quad(i=1,2, \cdots, n)
$$

the potential function becomes a function of $x=\left(x_{1}, x_{2}, \cdots, x_{n}\right)$ where the $x_{i}$ are orthogonal coordinates in $E^{n}$, and the directions of the $x_{i}$ and $q_{i}$, and hence the origins of the $x$ and $q$-systems, coincide. Then, for every motion of fixed energy level $h$, we define as the configuration space of the motion the closed subset $\Phi(h)$ in $E^{n}$ defined by

$$
\Phi(h)=\{x: U(x)+h \geq 0\} .
$$

The line element in $\Phi$ is the Euclidean arc length $d s$, defined by

$$
d s^{2}=d x_{i} d x_{i}
$$

(where the double index summation convention is used, as will be done throughout).

Jacobi's form of the principle of least action (valid for simple systems) is

$$
\delta A=\delta \int_{P_{1}}^{P_{2}}(2(U+h))^{1 / 2}\left(d x_{i} d x_{i}\right)^{1 / 2}=0,
$$

where $P_{1}$ and $P_{2}$ are distinct points in $\Phi$.

It is of immediate and practical importance to point here to the meaning of the symbol $\delta$. The principle of least action states that the trajectory is that curve for which the action integral in (5) is stationary among all curves joining $P_{1}$ and $P_{2}$ which lie in the open neighborhood of the trajectory. In fact, the existence of such an open neighborhood is presupposed in the derivation of the Euler equations of (5). Consider, for instance, the case where $P_{1}$ and $P_{2}$ are both on $\Gamma$, and consider a curve joining $P_{1}$ and $P_{2}$, also lying on $\Gamma$. This curve actually makes the action integral an absolute minimum; but it cannot be a trajectory because no open neighborhood exists around such a curve.

We may now construct a Riemann space $R^{n}$ on the open subset of $E^{n}$ defined by

$$
\Theta(h)=\{x: U(x)+h>0\}
$$

whose line element is defined by

$$
d A^{2}=2(U+h) d x_{i} d x_{i}
$$

The subset $\Theta$ is the largest open subset of $E^{n}$ surrounded by $\Gamma$, and it is simply connected because $\Gamma$ is a closed, connected hypersurface enclosing the origin. Moreover, $d A^{2}$ is positive, definite in the $d x_{i}$. Hence, the theorems of Riemannian geometry may be applied in $\Theta$. 
Representations of the motion. The integrand in (5) is a first order homogeneous function of the $d x_{i}$. One deduces that the functional $A$ depends on the path, but the path may be parametrized in many ways, and the value of $A$ is independent of the choice of parameter [4]. Let $\alpha$ be a parameter; then, one may write (5) as

$$
\delta \int_{\alpha\left(P_{1}\right)}^{\alpha\left(P_{2}\right)}(2(U+h))^{1 / 2}\left(x_{i}^{\prime} x_{i}^{\prime}\right)^{1 / 2} d \alpha=0,
$$

where primes denote differentiation with respect to $\alpha$. Different choices of the parameter result in different representations of the motion.

When $\alpha$ is the time $t$, the Euler equations of (8) are the equations of motion

$$
\ddot{x}_{i}=\partial U(x) / \partial x_{i}, \quad(i=1,2, \cdots, n)
$$

where use was made of the energy integral

$$
2(U+h)=\dot{x}_{i} \dot{x}_{i} .
$$

When $\alpha$ is the Euclidean arc length $s$, defined in (4), the Euler equations of (8) are

$$
2(U+h) x_{i}^{\prime \prime}+\left(\left(\partial U / \partial x_{i}\right) x_{i}^{\prime}\right) x_{i}^{\prime}-\partial U / \partial x_{i}=0, \quad(i=1,2, \cdots, n),
$$

where use was made of

$$
x_{i}^{\prime} x_{i}^{\prime}=1 .
$$

When $\alpha$ is the Riemann arc length $A$, defined in (7), the Euler equations of (8) become

$$
2(U+h) x_{i}^{\prime \prime}+2\left(\left(\partial U / \partial x_{i}\right) x_{i}^{\prime}\right) x_{i}^{\prime}-\left(x_{i}^{\prime} x_{i}^{\prime}\right)\left(\partial U / \partial x_{i}\right)=0, \quad(i=1,2, \cdots, n),
$$

where use was made of

$$
2(U+h) x_{i}^{\prime} x_{i}^{\prime}=1 .
$$

One may regard (9) as the Newtonian equations of motion of a particle having unit mass, moving in the $n$-dimensional configuration space under the force

$$
\nabla U=\left(\partial U / \partial x_{1}, \partial U \partial x_{2}, \cdots, \partial U / \partial x_{n}\right) .
$$

This system of the unit particle in $n$-space is called the pseudo-system. When it moves in the configuration space it traces out a trajectory, and (11) are the differential equations of that trajectory. Finally, (13) are the differential equations of this trajectory in the $n$-dimensional Riemann space $\Theta$, expressed in terms of the Riemannian arc length $A$.

It is evident that choices of a parameter other than $t, s$, or $A$ are admitted. For instance, in the two-dimensional problem it was found convenient to choose one of the $x_{i}$ as a parameter [5].

The central theorem. We shall demonstrate the existence of equiperiodic $x_{i}(t)$ satisfying (9) which are such that, at some instant $t_{0}$, every $x_{i}\left(t_{0}\right)=0$, and at some other instant $t_{1}$, every $\dot{x}_{i}\left(t_{1}\right)=0$. These are well-known properties of the normal mode vibrations of linear systems, and they have been called vibrations-in-unison [1]. It is clear that, at $t=t_{0}$, the trajectory of a vibration-in-unison passes through the origin of $E^{n}$, and at $t=t_{1}$, it reaches the bounding surface. Then, one can show easily that, in consequence of the symmetry condition on $U$, this trajectory will reach the bounding surface again. Therefore, the trajectory of a vibration-in-unison is of the type called by 
Cooke and Struble BOB, and which has been called elsewhere [6] a simple trajectory.

The essential steps in the proof of the existence theorem are outlined below:

(a) We shall define, in the open set $\Theta$, a family of surfaces, called constant action surfaces, denoted by $\Gamma(A)$; these will be regarded as the images of the bounding surface under maps $Z^{A}: \Gamma \rightarrow \Gamma(A)$. For any fixed $A$, these maps are of class $C^{2}$, everywhere onto, and almost everywhere one-to-one.

(b) Every constant action surface is connected, symmetric with respect to the origin, and smooth everywhere except at those points for which the maps $Z^{A}$ are not one-to-one.

(c) All trajectories starting from $\Gamma$ intersect every $\Gamma(A)$ orthogonally for all $A \geq 0$.

(d) We shall show that there exists an $\alpha>0$ such that $\Gamma(A)$ is smooth everywhere if $0<A<\alpha$. One of these smooth constant action surfaces $\Gamma\left(A_{1}\right), 0<A_{1}<\alpha$ will be called the $S$-surface.

(e) Our problem will be formulated as a variational problem with variable endpoint by regarding the $S$-surface as the end-surface. It will be shown that, for the functional (5), the transversality condition is identical with orthogonality, and that the Weierstrass and Legendre conditions are satisfied everywhere except on $\Gamma$.

(f) Finally, a Riemann metric is defined on the closed region surrounded by the $S$-surface. It will be shown that this region is compact in the sense of this metric, and that any point in this region may be joined to the $S$-surface by a shortest join which is at least as short in terms of the Riemann length as any other curve joining that point to the $S$-surface. Therefore, this shortest arc satisfies the Euler equations and the transversality condition. Since the origin is one of the points of the region surrounded by $S$, the proof is complete.

Constant action surfaces. Consider the unit mass of the pseudo-system starting from an initial point $X$ on $\Gamma$. Since the gradient vector $\nabla U$ does not vanish on $\Gamma$ (admissibility condition (iii)) the unit mass moves into the open region $\Theta$ defined by (6). But on $\Gamma$ the velocity vector $\dot{x}=\left(\dot{x}_{1}, \dot{x}_{2}, \cdots, \dot{x}_{n}\right)$ vanishes; in consequence, the initial conditions on the equations of motion are completely determined by $X$. Now, the equations of motion satisfy easily the conditions for existence and uniqueness of solutions; i.e. their right-hand sides satisfy a uniform Lipschitz condition throughout $\Phi$ (the Lipschitz condition follows from the smoothness condition on $U$, and the uniformity is a consequence of the existence of the energy integral for all $t \geq 0$ ). Hence, there exists a unique solution of (9) for all $t \geq 0$ which will be denoted by

$$
\varphi(t, X) \triangleq \varphi_{t}(X) .
$$

If we call $x(t, X)$ and $\dot{x}(t, X)$ the first-half and the second-half components of $\varphi_{t}(X)$, respectively, then $x(t, X)$ is the configuration at $t$, and $\dot{x}(t, X)$ is the velocity vector at that configuration.

Along the trajectory, Hamilton's form of the action integral is

$$
A=\int_{0}^{t}\|\dot{x}(s, X)\|^{2} d s
$$

where the symbol $\|:\|$ denotes the Euclidean norm. The integrand in (15) is twice the kinetic energy and, thus, positive everywhere in $\Theta$. Since the trajectory moves into that region, the action is a nonnegative, increasing function of time, or

$$
\partial A / \partial t=\|\dot{x}\|^{2}>0 .
$$


It follows that, for any preassigned, fixed value of the action $A>0$, one can always find a unique time $t_{\boldsymbol{A}}$ such that (15) is satisfied. Expressed differently, for every trajectory starting at $X$ on $\Gamma$ when $t=0$, there exists a unique configuration $x\left(t_{A}, X\right)$ such that the action has the preassigned value $A$. We shall call $x\left(t_{A}, X\right)$ the extremity of the trajectory.

Now, the action $A$ is of class $C^{2}$ in $X$ and $C^{4}$ in $t$, and it is an increasing function of $t$ by (16). Therefore, one may apply the implicit function theorem to obtain the time at which the action along the trajectory has the value $A$. We denote the time at which the trajectory reaches the extremity $x\left(t_{A}, X\right)$ by

$$
t_{A} \triangleq \Psi_{A}(X) .
$$

This function is of class $C^{2}$ in $X$, and $C^{4}$ in $A$ everywhere in $\Theta$, and everywhere in $\Phi$ except on $\Gamma$. We now define a map

$$
Z^{A}: \Gamma \rightarrow \Theta \subset L^{n}
$$

given by

$$
Z^{A}(X)=x\left(t_{\boldsymbol{A}}, X\right) .
$$

Thus, $Z^{A}$ maps the initial point $X$ onto the extremity when the action is $A$. This map is of class $C^{2}$ in $X$ and $C^{4}$ in $A$, except on the bounding surface. It is interesting to observe that the map $Z^{A}$ is, in fact, the solution to the Riemann trajectory equations (13) because $A$ is the independent variable in these equations.

Finally, we define the constant action surface as

$$
\Gamma(A)=Z^{A}(\Gamma)=\left\{Z^{A}(X): X \in \Gamma\right\} .
$$

Expressed in words, $\Gamma(A)$ is the set of extremities of all trajectories starting from $\Gamma$ such that the action integral has the same value $A$ along all of them. Thus, a constant action surface is the image of $\Gamma$ under the map $Z^{A}$. Since $\Gamma$ is a closed hypersurface, $\Gamma(A)$ is also a closed hypersurface. Moreover, $\Gamma$ is smooth; hence, $\Gamma(A)$ is smooth in the neighborhood of every point for which the map $Z^{A}$ is one-to-one. We now state

Lemma 1. There exists a small $A_{0}>0$ such that, for every positive $A<A_{0}$, the surfaces $\Gamma(A)$ are locally $(n-1)$-dimensional everywhere. Hence the map $Z^{A}$ is locally one-to-one for all positive $A<A_{0}$.

In the proof we make use of the following result from matrix theory: In a Euclidean space $E^{n}$, the null space of an $(n \times n)$ matrix $B$ is a linear subspace of $E^{n}$, generated by all vectors $a$ for which $B a=0$. The dimension of the null space is the maximum number of linearly independent vectors in the null space, and it is equal to $n$ minus the rank of $B$. If $B$ has the rank $\alpha$, and if $a_{1}, a_{2}, \cdots, a_{n}$ are linearly independent vectors for which $a_{\alpha+1}, a_{\alpha+2}, \cdots, a_{n}$ are in the null space of $B$, then the vectors $B a_{i},(j=1,2, \cdots, \alpha)$ are linearly independent; thus, they span the range space of $B$, that space being an $\alpha$-dimensional subspace of $E^{n}$.

To prove Lemma 1, we consider two trajectories starting, respectively, from neighboring points $X$ and $X+\delta X$ on $\Gamma$. The extremities of these trajectories, for a fixed $A$, are $Z^{A}(X)$ and $Z^{A}(X+\delta X)$. If we denote by $\delta Z_{i}$ the $i$ th component of the difference vector

$$
\delta Z=Z^{\Lambda}(X+\delta X)-Z^{\Lambda}(X)
$$


that component is

$$
\delta Z_{i}=\dot{x}_{i}\left(t_{A}, X\right) \delta t_{A}+\frac{\partial x_{i}\left(t_{A}, X\right)}{\partial X_{k}} \delta X_{k}
$$

where $t_{A}$ is defined implicitly by the line integral along the trajectory

$$
A=\int_{0}^{t_{A}}\|\dot{x}(s, X)\|^{2} d s=\int_{0}^{t_{A}^{A}} 2[h+U(x(s, X))] d s .
$$

But, since the action is the same along both trajectories, one has from the first equality (22)

$$
\left\|\dot{x}\left(t_{A}, X\right)\right\|^{2} \delta t_{A}+\int_{0}^{t_{A}} 2 \dot{x}_{i}(s, X) \frac{\partial \dot{x}_{i}(s, X)}{\partial X_{k}} d s \delta X_{k}=0
$$

and from the second,

$$
\left\|\dot{x}\left(t_{A}, X\right)\right\|^{2} \delta t_{A}+\int_{0}^{t_{A}} 2 \ddot{x}_{i}(s, X) \frac{\partial x_{i}(s, X)}{\partial X_{k}} d s \delta X_{k}=0,
$$

where use was made in (26) of the equation of motion $\partial U / \partial x_{i}=\ddot{x}_{i}$. If one integrates the integral in (24) by parts and adds the resulting equation to (23), there results

$$
\left\|\dot{x}_{i}\left(t_{A}, X\right)\right\|^{2} \delta t_{A}+\dot{x}_{i}\left(t_{A}, X\right) \frac{\partial x_{i}\left(t_{A}, X\right)}{\partial X_{k}} \delta X_{k}=0 .
$$

Then, comparing (21) and (25) one has the important result

$$
\dot{x}_{i}\left(t_{A}, X\right) \delta Z_{i}=0
$$

or the inner product of velocity vector and difference vector vanish. This means that the velocity vector $\dot{x}$ is orthogonal to $\Gamma(A)$ for every trajectory originating at any point $X$ on $\Gamma$. In other words, the constant action surfaces are the geodesic parallels of the trajectories. This remarkable result is due to Thomson and Tait [7]. Since $2(U+h)=$ $\|\dot{x}\|^{2}>0$, one has from (25)

$$
\delta t_{A}=-\frac{\dot{x}_{i}\left(t_{A}, X\right)}{\|\dot{x}\|^{2}} \frac{\partial x_{i}\left(t_{A}, X\right)}{\partial X_{k}} \delta X_{k}
$$

and the substitution of this quantity in (21) gives

$$
\begin{aligned}
\delta Z_{i} & =-\frac{\dot{x}_{i} \dot{x}_{i}}{\|\dot{x}\|^{2}} \frac{\partial x_{i}}{\partial X_{k}} \delta X_{k}+\frac{\partial x_{i}}{\partial X_{k}} \delta X_{k} \\
& =\left(\delta_{i i}-\frac{\dot{x}_{i} \dot{x}_{i}}{\|\dot{x}\|^{2}}\right) \frac{\partial x_{i}}{\partial X_{k}} \delta X_{k}
\end{aligned}
$$

where $\delta_{i i}$ is Kronecker's delta, and $\dot{x}_{i},\|\dot{x}\|^{2}$ and $\partial x_{i} / \partial X_{k}$ are all evaluated at $t_{-}=t_{A}$. The matrix form of (27) is

$$
\left[\begin{array}{c}
\delta Z_{1} \\
\vdots \\
\delta Z_{n}
\end{array}\right]=B_{A}(X)\left[\begin{array}{c}
\delta X_{1} \\
\vdots \\
\delta X_{n}
\end{array}\right]
$$


where $B_{A}(X)$ is the $(n \times n)$ matrix

$$
B_{A}(X)=\left[\begin{array}{cccc}
1-\frac{\dot{x}_{1}^{2}}{\|\dot{x}\|^{2}} & -\frac{\dot{x}_{1} \dot{x}_{2}}{\|\dot{x}\|^{2}} & \cdots & -\frac{\dot{x}_{1} \dot{x}_{n}}{\|\dot{x}\|^{2}} \\
-\frac{\dot{x}_{2} \dot{x}_{1}}{\|\dot{x}\|^{2}} & 1-\frac{\dot{x}_{2}^{2}}{\|\dot{x}\|^{2}} & \cdots & -\frac{\dot{x}_{2} \dot{x}_{n}}{\|\dot{x}\|^{2}} \\
-\frac{\dot{x}_{n} \dot{x}_{1}}{\|\dot{x}\|^{2}} & -\frac{\dot{x}_{n} \dot{x}_{2}}{\|\dot{x}\|^{2}} & \cdots & 1-\frac{\dot{x}_{n}^{2}}{\|\dot{x}\|^{2}}
\end{array}\right]\left[\begin{array}{ccc}
\frac{\partial x_{1}}{\partial X_{1}} & \cdots & \frac{\partial x_{1}}{\partial X_{n}} \\
\vdots & \cdots & \frac{\partial x_{2}}{\partial X_{n}} \\
\frac{\partial x_{n}}{\partial X_{1}} & \cdots & \frac{\partial x_{n}}{\partial X_{n}}
\end{array}\right]
$$

and this matrix is of class $C^{1}$ in $X$ and $C^{3}$ in $A$.

But $\delta X$ and $\delta Z$ are vectors of infinitesimal length. The first lies in the tangent plane of $\Gamma$ at $X$, and the second in the tangent plane of $\Gamma(A)$ at $Z^{A}(X)$. Hence, the matrix $B_{A}(X)$ is a map which assigns a small neighborhood of the tangent plane of $\Gamma$ at $X$, to a small neighborhood of the tangent plane of $\Gamma(A)$ at $Z^{A}(X)$. Now, the matrix is the product of two $(n \times n)$ matrices. The first of these is symmetric, and its eigenvalues are all unity except for one which is zero. Therefore, its null space is one-dimensional and it is, in fact, in the direction tangent to the trajectory. The second matrix of (28) is an $(n \times n)$ identity matrix when $t=0$; i.e. when $A=0$. Therefore, for $A=0, B_{A}(X)$ is of rank $n-1$, and its null space is orthogonal to $\Gamma$ because any trajectory originating on $\Gamma$ is orthogonal to it [1]. But $B_{A}(X)$ is of class $C^{3}$ in $A$. Therefore, for $A$ sufficiently small, $B_{A}(X)$ remains of rank $n-1$, and its null space is in the neighborhood of the gradient $\nabla U$ at $X$ on $\Gamma$, and therefore not in the tangent plane of $\Gamma$ at $X$. This implies that, for sufficiently small $A$, the tangent plane of $\Gamma(A)$ at $Z^{A}(X)$ is also $(n-1)$-dimensional, thus proving Lemma 1 . As a consequence of Lemma 1 , we prove

Lemma 2. There exists a positive number $\alpha$ such that, if $0 \leq A<\alpha$, the map $Z^{A}$ is one-to-one, which implies that $\Gamma(A<\alpha)$ is a smooth closed hypersurface.

To prove this lemma, we shall show that there exists a small positive $A$ such that $Z^{A}(X)$ is globally one-to-one. Consider two trajectories issuing, respectively, from nonneighboring points $X^{\prime}$ and $X^{\prime \prime}$ on $\Gamma$, and let us suppose that they have the same extremity when $A=A_{1}$, or

$$
x=Z^{A_{2}}\left(X^{\prime}\right)=Z^{A_{2}}\left(X^{\prime \prime}\right) .
$$

In other terms, $Z^{A_{2}}(X)$ is not one-to-one at $x$. Then it follows from the smoothness of $\Gamma$, and from the fact that the trajectories are orthogonal to $\Gamma$ at $X^{\prime}$ and $X^{\prime \prime}$, respectively, that $x$ is not near the bounding surface. Hence, there exists a least upper bound $A=A^{\prime \prime}$ such that $Z^{A^{\prime \prime}}$ is globally one-to-one, and we have shown that there exists also a least upper bound $A^{\prime}$ such that $Z^{A^{\prime}}$ is locally one-to-one. Then one chooses $\alpha$ to be the lesser of $A^{\prime}$ and $A^{\prime \prime}$, which proves Lemma 2 .

Properties of trajectories. In order to demonstrate the existence of normal mode vibrations, it is necessary to make use of certain properties of trajectories. Some of these are given here.

One of the properties, demonstrated elsewhere [5] is

Property 1. Every trajectory which intercepts the bounding surface $\Gamma$, intercepts it orthogonally. 
Property 2. When a trajectory, starting from a configuration $x$ in $\Theta$, intercepts the bounding surface $\Gamma$, it will return to $x$ along the same path on which it reached $\Gamma$.

It is clear that such a property, if it exists, is a consequence of uniqueness theorems. We shall make the demonstration of Property 2 in some detail because, in proving it, we deduce certain results, needed later on.

It is convenient to write the equations of motion (9) in the form

$$
d y / d t=f(y),
$$

where $f$ is a $2 n$-dimensional vector having components

$$
f_{i}=\dot{x}_{i}, \quad f_{n+i}=\partial U(x) / \partial x_{i}, \quad(i=1,2, \cdots, n)
$$

and $y(x, \dot{x})$ is a $2 n$-dimensional vector whose first $n$ components are the configurations $x$, and the second $n$ components are the components of the velocity vector $\dot{x}$ at $x$; hence the $y$-space is the phase space.

As stated earlier, the right-hand sides of (29) satisfy a uniform Lipschitz condition. Thus, there exists a unique solution of (29)

$$
y(t)=\varphi_{t}(Y), \quad|t| \leq \infty
$$

satisfying

$$
y(0)=\varphi_{0}(Y)=Y
$$

where $Y=(X, \dot{X})$, and $X$ is in $\Phi$.

The quantity $t$ in (31) plays the role of a parameter of the transformation $\varphi_{t}$ which maps the initial point at $t=0$ into a terminal point $(x, \dot{x})$ at $t$.

Because of the smoothness condition $(i)$ on $U, f(y)$ is of class $C^{2}$; i.e. the transformation $\varphi_{t}$ is of class $C^{2}$ in $Y$ and $C^{3}$ in $t$ [8]. Moreover, since (29) is autonomous, $\varphi_{t}$ is a one-parameter group of transformations satisfying

$$
\varphi_{t+\mathfrak{s}}=\varphi_{t} \circ \varphi_{\mathrm{s}}
$$

where the small circle denotes the composition of two transformations. Expressed differently, a terminal point $y(s+t)$ is, on the one hand, the image of an initial point $Y$ at $t=0$ mapped by $\varphi_{t+t}$, and on the other, it is the image of $\varphi_{t}(Y)$ mapped by $\varphi_{1}$, where $\varphi_{t}(Y)$ is the image of $Y$ mapped by $\varphi_{t}$.

From the uniqueness of solutions of (29) and from (32), it follows that no two distinct trajectories of the same energy level can have a common tangent in the configuration space.

It is a property of the one-parameter group of transformations that the inverse transform exists and is given by

$$
\varphi_{t}^{-1}=\varphi_{-1}
$$

Suppose we write (29) in the two forms

$$
d x_{i} / d t=\dot{x}_{i}, \quad d \dot{x}_{i} / d t=\partial U / \partial x_{i}
$$

with $x(0)=X, \dot{x}(0)=\dot{X}$, and

$$
d x_{i} / d(-t)=-\dot{x}_{i}, \quad d\left(-\dot{x}_{i}\right) / d t=\partial U / \partial x_{i}
$$

with $x(0)=X, \dot{x}(0)=-\dot{X}$. Then, if the solution of the first set is

$$
(x, \dot{x})=\varphi_{\imath}(X, \dot{X}),
$$


that of the second is

$$
(x,-\dot{x})=\varphi_{-t}(X,-\dot{X}) .
$$

In particular, if $X$ is on $\Gamma$, one has $\dot{X}=0$, so that

$$
(x, \dot{x})=\varphi_{1}(X, 0) ; \quad(x,-\dot{x})=\varphi_{-t}(X .0) .
$$

In consequence, when $\dot{x}(0)=0$,

$$
(x, \dot{x})=\varphi_{2 t}(x,-\dot{x}),
$$

which proves Property 2.

As a corollary of Property 2, one also has

Property 3. If a trajectory which issues from a point $X^{\prime}$ on $\Gamma$ intersects $\Gamma$ at some other point $X^{\prime \prime} \neq X^{\prime}$ on $\Gamma$, it is a closed trajectory.

Clearly, this property holds for systems whether they satisfy the symmetry condition (ii) on $U$, or not. When that condition is satisfied as well, trajectories also have

Property 4. If a trajectory, starting from $X$ on $\Gamma$, passes through the origin of the configuration space, it also intercepts the point $-X$ on $\Gamma$.

This is a consequence of

Property 5. Every trajectory which passes through the origin of the configuration space is symmetric with respect to it.

This property was first given by Mawhin [9]. One of the properties which is central to our proof of the existence of simple trajectories is

Property 6. All trajectories starting from the bounding surface $\Gamma$ intersect every constant action surface orthogonally.

This property was demonstrated in (26). It is a general property of all families of trajectories, whether they issue from points on a fixed surface, or from a fixed point in different directions.

Local minimization. By Lemma 2, there is a constant action surface $\Gamma\left(A_{1}\right), 0<$ $A_{1}<\alpha$, given by

$$
\Gamma\left(A_{1}\right)=Z^{A_{1}}(\Gamma)=\left\{Z^{A_{1}}(X): X \in \Gamma\right\}
$$

on which the map $Z^{A_{1}}$ is one-to-one everywhere; we call this surface the $S$-surface. It lies near to, and resembles in every way, the bounding surface $\Gamma$; i.e. it is a closed, connected, smooth surface which is symmetric with respect to the origin of $E^{n}$. Let $M$ be the closed region surrounded by the $S$-surface, and let $M^{0}$ be the interior of $M$. Then, the open set $M^{0}$ is also connected, and the origin is one of the points of $M^{0}$. It is evident that each pair of points in $M$ can be connected by a curve of finite Euclidean length lying entirely in $M$. In particular, every point of $M^{0}$ can be connected to all points of $S$ by such a curve.

Let $C_{x y}$ be a curve connecting two points $x$ and $y$ in $M$. Then, the Riemann length of $C_{x y}$, denoted by $A\left(C_{x y}\right)$, is the action integral evaluated along $C_{x y}$. Since $M$ is closed and bounded, and since the potential function $U$ is continuous, there exists a maximum value $\bar{m}$ of $(2(U+h))^{1 / 2}$ on $M$. Consequently, any pair of points in $M$ can also be connected by a curve of finite Riemann length. 
Consider a curve $C_{X x}$ joining $x \in M$ to a point $X \in S$ and a small open neighborhood $\mathfrak{X}$ around $C_{X x}$. While we do not insist that $\mathfrak{X}$ lie necessarily entirely in $M$, we do require that $U(y)+h>0$ for all points $y$ in $\Re$; this is done in order that the variational problem (5) be meaningful. We seek a curve $C_{X x}$ such that

$$
A\left(C_{X x}\right)<A\left(C_{Y x}\right)
$$

for any curve $C_{Y x} \subset \Re$ different from $C_{X x}$, where $Y \in \Re \cap S$. A curve $C_{X x}$ satisfying (34) will be called a locally minimizing arc. The word "local" implies that all curves neighboring on $C_{X x}$ are admitted to the competition.

This is a problem in the calculus of variations with movable endpoint; hence, the locally minimizing arc must satisfy all necessary conditions; i.e.

(I) the Euler equations,

(II) the transversality condition,

(III) the Weierstrass and Legendre conditions,

(IV) Jacobi's focal point condition.

It was already shown in the section "Representations of the motion" that whenever a curve satisfies condition (I), it is a trajectory. We shall now show that the transversality condition is identical with orthogonality in the sense of $E^{n}$. In fact, after some calculation, the transversality condition for the functional (5) reduces to

$$
(2(U+h))^{1 / 2} x_{i}^{\prime} d x_{i}=0,
$$

where $x^{\prime}=\left(x_{1}^{\prime}, x_{2}^{\prime}, \cdots, x_{n}^{\prime}\right)$ is the unit tangent vector of the trajectory, and $d x=$ $\left(d x_{1}, d x_{2}, \cdots, d x_{n}\right)$ is an arbitrary vector in the tangent plane of the $S$-surface. Since$U+h>0$ on $S$, (35) implies that every locally minimizing arc is orthogonal to $S$; hence, by Property 6 , it is an arc of a trajectory starting from a point on $\Gamma$.

The Weierstrass and Legendre conditions may be readily verified for the functional (5). In fact, the Weierstrass $E$-function may be written in the form

$$
E\left(x, x^{\prime}, X^{\prime}\right)=(2(U+h))^{1 / 2}\left(\left\|X^{\prime}\right\|\left\|x^{\prime}\right\|-x_{i}^{\prime} X_{i}^{\prime}\right)
$$

and, by the Schwarz inequality, $E>0$ whenever two vectors $X^{\prime}$ and $x^{\prime}$ are linearly independent and $U+h>0$. Thus, the Weierstrass condition is satisfied everywhere except on the bounding surface $\Gamma$. In a similar manner, Legendre's condition is easily verified. The focal point condition (IV) will not be needed in the existence proof of a simple trajectory; hence it is not examined.

The shortest join. In this section, we restrict our treatment to the closed region $M$ surrounded by the $S$-surface; our interest centers on a shortest join (not necessarily a trajectory) between any pair of points in $M$. We shall define the Riemann distance between any pair of points $x$ and $y$ in $M$ by the distance function

$$
d(x, y)=\inf A\left(C_{x y}\right),
$$

where inf refers to all curves lying in $M$ and connecting $x$ and $y$. A curve $C_{x y}$ is said to be a shortest join between $x$ and $y$ if

$$
d(x, y)=A\left(C_{x y}\right) .
$$

One can readily demonstrate 
I.емма 3. The Riemann distance $d(x, y)$ satisfies all the axioms of a metric; i.e., (i) $d(x y) \geq 0$; (ii) $d(x, y)=0$ only when $x=y$; (iii) $d(x, y)=d(y, x)$; (iv) $d(x, y) \leq$ $d(x, z)+d(z, y)$.

and

Lemma 4. Let $\left\{x^{i}\right\},(i=1,2, \cdots)$ be a sequence of points in $M$. Then $\lim \left\|x-x^{i}\right\|=0$ if and only if $\lim d\left(x, x^{i}\right)=0$. Hence, the Riemann metric space $M$ is compact.

These lemmas follow from the definition (36) of $d(x, y)$ and from the inequality in $M$ :

$$
m\|x-y\| \leq d(x, y) \leq \bar{m}\|x-y\|
$$

where $\bar{m}$ is the largest value of $(2(U+h))^{1 / 2}$ and $m$ is the smallest value of this quantity in $M$.

As a consequence of Lemma 4, one has

I.EMma 5. Any pair of points in $M$ can be connected by a shortest join.

The proof of this lemma rests on the following

Lemma ([10]). If in a finitely compact set $H$, the points $x$ and $y$ can be connected by $a$ rectifiable curve, then there is a shortest join in $H$.

Here, the term "finitely compact set" means a set in which every infinite bounded subset has an accumulation point in the set, and a "rectifiable curve" is a parameterized curve $C: x(t)=\left(x_{1}(t), x_{2}(t), \cdots, x_{n}(t)\right)$ in which the range of the parameter $t$ is $0 \leq$ $t \leq \beta$ where $\beta$ is bounded.

Evidently, a compact set is finitely compact. In particular, the Riemann space $M$ is finitely compact, and any pair of points in $M$ can be connected by a curve of finite Riemann length; thus Lemma 5 is true.

It is noted that a shortest join is not necessarily a trajectory because the existence of an open neighborhood about that curve is not assured. For instance, if $X$ and $Y$ are points on the $S$-surface, and if the shortest join $C_{X Y}$ lies on $S$ (because the function $U(x)+h$ may take on lesser values on $S$ than on the interior $M^{0}$ ), no open neighborhood exists about $C_{X Y}$; in consequence, the variational problem (5) may, then, not be meaningful.

Existence proof. Here we prove

THEOREM 1. Every point in the configuration space can be connected to the bounding surface $\Gamma$ by a trajectory whose Riemann length is at least as short as that of any other curve connecting that point to $\Gamma$.

The Riemann distance between a point $x \in M$ and the $S$-surface is defined as

$$
d_{S}(x)=\inf \{d(x, X): X \in S\} .
$$

Let $x$ be an interior point of $M$. Then, a curve $C_{X x}$ connecting $x \in M^{0}$ to $X \in S$ is said to be an S-min-arc if

$$
d_{S}(x)=A\left(C_{X x}\right)
$$

Since $S$ is a closed subset of $M, S$ is compact; therefore, for any point $x \in M^{0}$ there is a point $X \in S$ such that

$$
d_{S}(x)=d(x, X)
$$


But, by Lemma 4 , there is a shortest join $C_{X x}$ between $x$ and $X$, so that $d_{S}(x)=A\left(C_{X_{x}}\right)$. Hence, an $S$-min-arc exists for any point $x \in M^{0}$.

Now, it is evident that every $S$-min-arc lies entirely in $M^{0}$ except for its endpoint on $S$. Hence, there does exist an open neighborhood $\mathfrak{N}$ around each $S$-min-arc for which (5) is meaningful. It follows that an $S$-min-are satisfies all conditions necessary for it to be a locally minimizing arc as well. In particular, it satisfies the Euler equations and the transversality condition. Thus, it is a trajectory originating from the bounding surface $\Gamma$, which proves Theorem 1 .

As a corollary of Theorem 1, one has

Theorem 2. In every admissible system having many degrees of freedom, there exists at least one simple trajectory.

A simple trajectory connects the origin of $E^{n}$ with $\Gamma$. Since the origin is an interior point of $M$, Theorem 1 is applicable and Theorem 2 follows.

The simple trajectory whose existence has been demonstrated is the curve of shortest Riemann length between the origin and the bounding surface $\Gamma$. In the linear problem, this particular simple trajectory corresponds to the normal mode vibration for which the natural frequency is the highest. To show this, let $x_{1}, x_{2}, \cdots, x_{n}$ be the normal coordinates, and let $\omega_{i}$ be the natural frequency corresponding to $x_{i}$. For this problem, the bounding surface is

$$
\Gamma=\left\{X \in E^{n}: \frac{1}{2} \sum_{i=1}^{n} \omega_{i}^{2} X_{i}^{2}-h=0\right\}
$$

and the motion starting from a point $X=\left(X_{1}, X_{2}, \cdots, X_{n}\right) \in \Gamma$ is

$$
x_{i}=X_{i} \cos \omega_{i} t, \quad(i=1,2, \cdots, n) .
$$

Along the trajectory corresponding to this motion, the action is

$$
A=\frac{1}{2} \sum_{i=1}^{n} \omega_{i}^{2} X_{i}^{2}\left(t-\frac{1}{2 \omega_{i}} \sin 2 \omega_{i} t\right)
$$

The $i$ th normal mode vibration is that for which $X_{i}=0$ for all $j \neq i$. The time $t_{i}$ required for the trajectory to reach the origin is $t_{i}=\pi / 2 \omega_{i}$. Hence, the corresponding action is $A_{i}=\left(\pi / 2 \omega_{i}\right) h$. Therefore, the action is least for the largest $\omega_{i}$.

Acknowledgment. Research sponsored by the Air Force Office of Scientific Research, Office of Aerospace Research, United States Air Force under AFOSR Grants No. 1057-66 and No. 1057-67.

\section{REFERENCES}

[1] R. M. Rosenberg, On nonlinear vibrations of systems with many degrees of freedom, Adv. Appl. Mech., vol. 9. Academic Press, New York, 1966, pp. 156-243

[2] C. H. Cooke and R. A. Struble, The existence of periodic solutions and normal mode vibrations in nonlinear systems, Quart. Appl. Math. 24, 177-193 (1966)

[3] Ta-Lun Yang, and R. M. Rosenberg, On the vibrations of a particle in the plane, Internat. J. Nonlinear Mech., 2, 1-25 (1967)

[4] G. A. Bliss, Lectures on the calculus of variations, 7th Impr., Univ. of Chicago Press, Chicago, Ill., 1963

[5] R. M. Rosenberg, On normal mode vibrations, Proc. Camb. Philos. Soc. 60, 595-611 (1964) 
[6] J. K. Kuo, On nonsimilar normal mode vibrations of nonlinear systems having many degrees of freedom, Ph.D. thesis, Univ. of Calif., Berkeley, 1964

[7] Sir William Thomson and P. G. Tait, Treatise on natural philosophy, vol. 1, p't. 1, new ed., Cambridge Univ. Press, 1879

[8] P. Hartman, Ordinary differential equations, Wiley, New York, 1964

[9] J. Mawhin, Oscillations en modes normaux des systèmes dynamiques nonlinéaires à plusieurs degrés de liberté, Bull. Soc. Royale Sciences Liège 9, 540-557 (1964)

[10] H. Busemann, The geometry of geodesics, Academic Press, New York, 1955, p. 25 